\title{
DESIGN OF FACE RECOGNITION SYSTEM USING PRINCIPAL COMPONENT ANALYSIS
}

\author{
Abin Abraham Oommen ${ }^{1}$, C.Senthil Singh ${ }^{2}$, M.Manikandan ${ }^{3}$ \\ ${ }^{I}$ M.Tech Student, Dept.of ECE, Toc H Institute of Science and Technology, Kerala, India \\ ${ }^{2}$ Associate Professor, Toc H Institute of Science and Technology, Kerala, India \\ ${ }^{3}$ Department of Electronics, Anna University, Chennai, India
}

\begin{abstract}
Face is considered to be one of the most important visual objects for identification. Recognition of human face is complex and it converts the face into a mathematical model. Face recognition is the most efficient and sophisticated method for the security systems. It is a biometric technology with a wide range of applications such as use in ATM machines, preventing voter's fraud, criminal identification, human computer interaction, etc. This paper describes the building of a face recognition system by using Principal Component Analysis method. PCA is the method for reduce the data dimension of the image. It is based on the approach that breaks the face images into a small set of characteristic feature images. These "eigenfaces" are the principal components of the initial data set of face images. Recognition is done by comparing the input face image with the faces in the data set through distance measuring methods. Here the face recognition system is developed using Matlab and it recognizes the input face from a set of training faces.
\end{abstract}

Keywords:Principal Component Analysis, eigenfaces, training faces

\section{INTRODUCTION}

A face recognition system is used for identifying or authenticatinga person from a digital image or a video frame from a video input [1]. The system tries to find the identity of a given face image according to the faces in the memory. Comparing face images is one of the many biometric ways of recognizing an individual. Applications of face recognition are widespread. It has many uses such as searching for an individual in a database, retrieving personal information about an individual, gaining access to data based on anyone's facial identity etc. It has many applications in human computer interaction, military bases, prevention of identity theft, airports, forensics, IT technologies, and financial services.

Face recognition mainly includes feature extraction, data reduction and recognition or classification [2]. Feature extraction is to find the most descriptive feature of the faces, making them to be easily distinguished from other faces. Data reduction is to reduce the dimension of the features but also not to destroy the most important information. It holds the basic information. Recognition is to select the distance measure method such as Euclidean distance that is used to classify the face images present in the database and input image. It is not possible to take the original data directly for processing, because the face image is with very high dimension. So it is important to select the effectively distinguished features for extraction and reduction. From all kinds of methods for the face recognition, Principal Component Analysis (PCA) is the effective method based on face as a global feature which considers the face as a single blob. It reduces the image dimension and at the same time primary information preserved.

In PCA, a training set is formed by collecting some face images. The eigenvectors of all the faces are calculated and the "eigenfaces" are the linear combination of these eigenvectors. Eigenfaces are the principal component of the data set consists of faces. Mathematically eigenfaces are the eigenvectors of the covariance matrix of the database. These eigenfaces or eigenvectors represents the significant variations among the face images. Weights of the eigenvectors are calculated for classification. When a new face is to be recognized, the Euclidian distance between test face and the database are calculated and are classified as face or non-face. Many numbers of experiments are done to evaluate the performance of face recognition system.

\section{EIGENVECTORS AND EIGENVALUES}

Eigenvector of a matrix is the vector such that, if that vector is multiplied with a matrix, it is equal to the integer multiple of that vector. And that integer value is denoted as the eigenvalue of the corresponding eigenvector. It can be explained by the equation,

$$
\mathrm{A} \times \mathrm{v}=\lambda \times \mathrm{v}
$$

Where $\mathrm{A}$ is the matrix, $\mathrm{v}$ is the eigenvector and $\lambda$ is the corresponding eigenvalue. 
Eigenvectors have some features which makes them suitable for the recognition purpose. Eigenvectors of a matrix is being the special matrix that is scaled by that matrix.Different matrices have different eigenvectors and only square matrices have eigenvectors.All the eigenvectors are perpendicular, i.e. at right angle to each other. In an $n \times n$ matrix, there are $n$ number of eigenvectors and corresponding eigenvalues.

\section{PRINCIPAL COMPONENT ANALYSIS}

PCA is the simplest of the true eigenvector based multivariate analysis. Mathematically, it is an orthogonal linear transformation that transforms the data to a new coordinate system. The use of Eigenfaces and vectors is commonly called as Principal Component Analysis. With PCA, the image must be used of same size and they are normalized to a particular size for the usage. In PCA, dimension of data is reduced and decomposes the face structure into orthogonal and uncorrelated components which are the eigenfaces. The face image can be represented as a weighted sum of the Eigenfaces. Mathematically, principal component analysis approach converts a set of correlated variables into a set of linearly uncorrelated variables. Each face image in the training set contributes to the eigenvectors. This can be displayed as an eigenface expresses the data in such a way as to highlight the similarities and differences. Eigenfaces are look like ghostly faces and each of them has some variations which deviate from the original image. If an eigenface with small eigenvalues are neglected, then the image will be a linear combination of reduced number of that faces.

When the new face image to be recognized its eigenvalue and weights are calculated. Then these weights are compared with the weights of the known face images in the training set. It is done by calculating the Euclidian distance between the new face image and the faces in training set. If the Euclidian distance is minimum, then the face is known and if it is maximum, then the face is unknown.

\subsection{Training Set}

A training set is formed by combining different face images. The images of all the different individuals were collected in a set called the training set. Face images are collected from different type of sources. The sources may be video camera or face image database on the photos. The collected face images should have the pose, illumination and expression, etc. variation in order to measure the efficiency of the face recognition system under these conditions. Processing of the face database require sometimes before it is used for recognition.

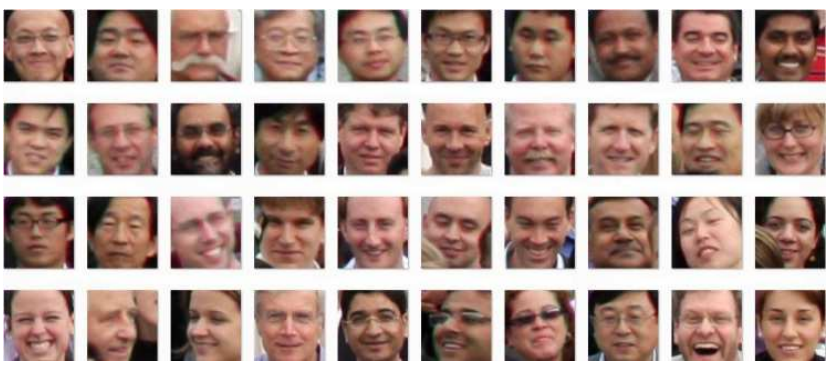

Fig-1: Example for training set

\subsection{Eigen Face}

Eigenfaces are the set of features in the form of vector that denotes the variation between faces. The basic idea behind eigenface is that think of the face as a weighted combination of some component or base faces. These components are the principal components of the face image. The training face images and the new face image can be represented as linear combination of eigenfaces. Each image in the training set has its own contribution on making the eigenfaces. So the eigenface represents the significant variation between all the faces. Eigenfaces are look likes the ghostly faces because some facial variations occur which deviates from the original image. Eigenvector with the largest eigenvalue has the most variation among the training vectors. And the eigenvector with small eigenvalue has the least variation. For generating eigenfaces, large set of images of human face is taken first. Then the images are normalized to get the features. Then the eigenvectors of the covariance matrix of the face image are extracted. Eigenvectors are formed by converting the image matrix into vector form and the vector should satisfy the eigenvalue equation. These eigenvectors are less in size but no data will be losses. Hence eigenfaces provide a means of applying data compression to images for identification purposes.

\subsection{Weight Vector}

For recognition, weight of the largest Eigenfaces is calculated from the training faces. When the new face image to be recognized, we calculate the weights associated with the Eigen faces, which linearly approximate the face or can be used to reconstruct the face. Now these weights are compared with the weights of the known face images so that it can be recognized as a known face or unknown face.

\subsection{Euclidian Distance}

In mathematics, the Euclidean distance or Euclidean metric is the ordinarydistance between two points that one would measure with a ruler, and is given by the Pythagorean formula. By using this formula as distance, Euclidean space (or even any inner product space) becomes a metric space. The associated norm is called the Euclidean norm. The Euclidean distance between point's $\mathrm{p}$ and $\mathrm{q}$ is the length of the line 
segment connecting them. Here we use the Euclidian distance to compare the training faces and input faces. We calculate the Euclidian distance between the input image and training face. Known face is with minimum Euclidian distance and unknown face with largest distance. The input face is considered to belong to a class if Euclidean distance ( $\varepsilon \mathrm{k}$ ) is below a threshold $\theta \varepsilon$. Then the face image is considered to be a known face. If the input image is above the threshold, the face is determined as unknown.

The Euclidian distance between two instances xi\&xj are given by

$$
\mathrm{d}(\mathrm{xi}, \mathrm{xj})=\sqrt{\sum_{\gamma=1}^{n}\left(\operatorname{ar}\left(x_{1}\right)-\operatorname{ar}\left(x_{j}\right)\right)^{2}}
$$

\section{EIGENFACE ALGORITHM}

The first step is to obtain a set $\mathrm{S}$ with $\mathrm{K}$ face images. Each image is converted into a vector of size $\mathrm{N}$ and the training set is formed with $\mathrm{K}$ faces is given by

$$
S=\left[\Gamma_{1}, \Gamma_{2}, \ldots \ldots, \ldots, T_{m}\right]
$$

Then obtain the mean of training faces as

$$
\Psi=\frac{1}{M} \sum_{i=1}^{M} T_{\mathrm{n}}
$$

And it is subtracted from the original face as

$$
\Phi_{\mathrm{i}}=\Gamma_{\mathrm{i}}-\Psi
$$

Eigen vectors are finding such that

$$
\lambda_{\mathrm{k}}=\frac{1}{M} \sum_{n=1}^{M}\left({u_{F i}{ }^{T}}_{\left.\Phi_{n}\right)^{Z}}\right.
$$

Whereu $\mathrm{k}_{\mathrm{k}}$ - eigen vector $\& \lambda_{\mathrm{k}}$ - eigen value

Covariance matrix is calculated as

$$
\mathrm{C}=\frac{1}{M} \sum_{n=1}^{M} \mathcal{F}_{n} \Psi_{n}{ }^{T}
$$

The new face is transformed into its Eigenface components as

$$
\omega_{\mathrm{k}}=u_{k}{ }^{2}(\Gamma-\Psi)
$$

Then the weight vector is formed by

$$
\Omega^{T}=\left[\omega_{1}, \omega_{2}, \omega_{3}, \ldots \ldots \omega_{\mathrm{m}}\right]
$$

The Euclidian distance between two instances $\mathrm{x}_{\mathrm{i}} \& \mathrm{x}_{\mathrm{j}}$ are given by

$$
\mathrm{d}\left(\mathrm{x}_{\mathrm{i}}, \mathrm{x}_{\mathrm{j}}\right)=\sqrt{\sum_{r=1}^{\mathrm{n}}\left(\operatorname{ar}\left(x_{i}\right)-\operatorname{ar}\left(x_{j}\right)\right)^{2}}
$$

Then we can calculate the Euclidian distance between our input face and training faces. It is given by

$$
\varepsilon_{\mathrm{k}}=\left\|\Omega-\Omega_{\pi_{\mathrm{L}}}\right\|
$$

If $\varepsilon_{\mathrm{k}}$ is below a threshold $\theta \mathrm{e}$, then the face is concluded to be known. If the input is above the threshold, then it's not a face.

\section{FLOW CHART}

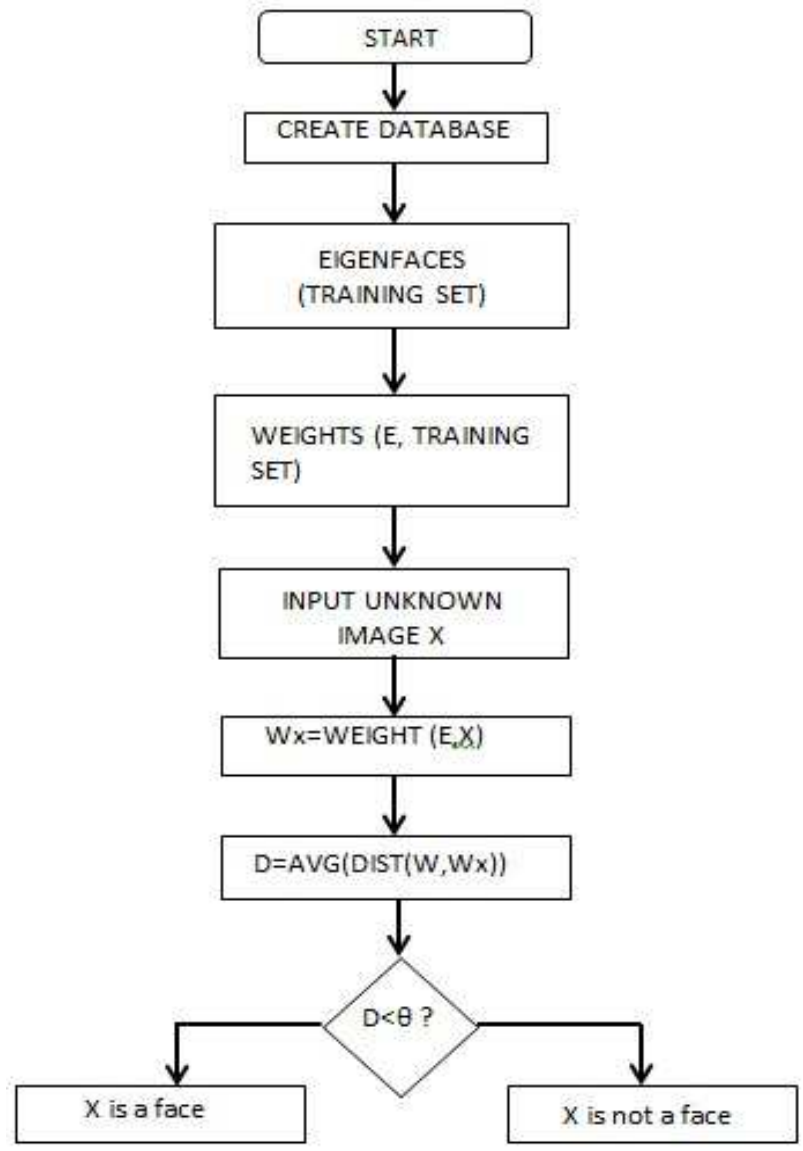

Fig -2: face recognition flow chart

\section{EXPERIMENTAL RESULTS}

The experiment is done using Matlab simulator. First the training set is created with face images. Here the training set consists of 500 face images. The face images are taken under normal light conditions. Varying conditions such as expression,pose and lighting has minimal effect on recognition process. The input face is read by using Matlab and is recognized from the training set and the total elapsed time is calculated. Figure shows the input image and recognized image. 
Input image is taken with size is lower than $10 \times 10$ and it is as shown in the figure 3 . Here we take the input image as low resolution image.

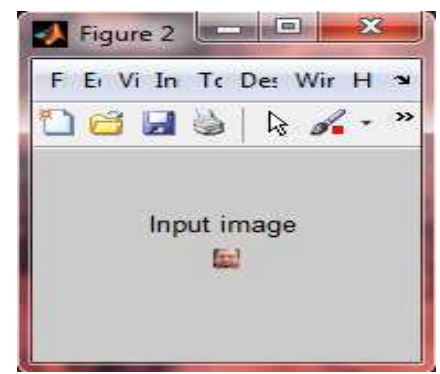

Fig -3: Input image

Then it is compared with the faces in the training set and selects the best match face. The recognized image is shown in figure 4 . It is of dimension of $480 \times 480$.

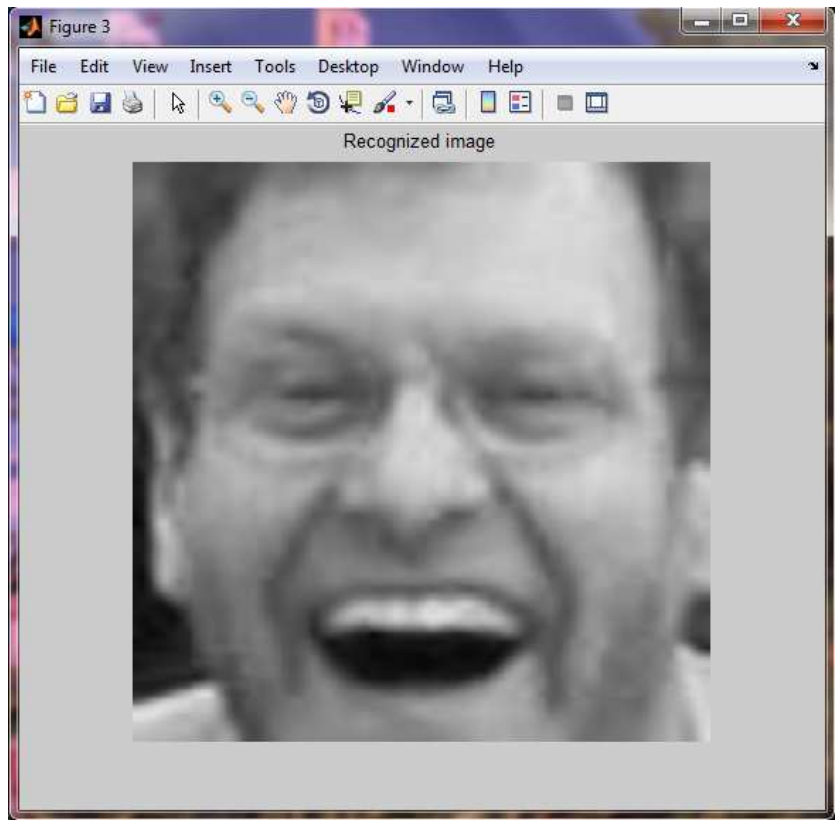

Fig -4: Recognized image

The output shows that the system takes a time of 63 seconds to recognize the input face from a training set of 500 images.

\section{CONCLUSIONS}

In this paper, the algorithm for recognizing the faces from an input is proposed. We are using the most flexible and efficient method for recognition, Principal Component Analysis. Here we took all the images in training set as a linear combination of weighted Eigen vectors. The system receives the input face and it is recognized from the training set. Recognition is done by finding the Euclidean distance between the input face and our training set. The system has been simulated using Matlab and it shows appreciable result and faster detection rate. The approach is definitely simple, easy and faster to implement.

\section{REFERENCES}

[1] Ali Irturk, Janarbek Matai and Ryan Kastner, "Design and Implementation of an FPGA-based Real-Time Face Recognition System". IEEE International Symposium on Field-Programmable Custom Computing Machines.2011.

[2] D.S.Chaudhari, SaurabhP.Bahurupi, "Principal Component Analysis for Face Recognition", International Journal of Engineering and Advanced Technology (IJEAT) June 2012.

[3] Matthew A. Turk and Alex P. Pentland. "Eigenfaces for recognisation". Journal of cognitive nerosciences, Volume 3, Number 1, Nov 27, 2002.

[4] H.T. Ngo, R. Gottumukkal, and V.K Asari, "A flexible and efficient hardware architecture for real-time face recognition based on eigenface", in IEEE Computer Society Annual Symposium on VLSI, 2005.

[5] I. Sajid and M.M Ahmed and SotiriosG.Ziavras, "Hardware-Based Speed Up of Face Recognition towards Real-Time Performance". 2010 13th Euromicro Conference on Digital System Design: Architectures, Methods and Tools

[6] A.Pentland and M. Turk, "Eigenfaces for recognition," Journal of Computer and System Sciences, vol. 3, no. 55, pp.119-139, 1991.

[7] Sanjay Kr Singh, AshutoshTripathi, AnkurMahajan, Dr $S$ Prabhakaran "Analysis of Face Recognition in MATLAB", International Journal of Scientific \& Engineering Research, Volume 3, Issue 2, February 20121 ISSN 2229-5518

[8] KandlaArora, "Real Time Application of Face Recognition Concept", International Journal of Soft Computing and Engineering (IJSCE) ISSN: 2231-2307, Volume-2, Issue-5, November 2012

[9] Senthilsingh and Manikandan, "Face Recognition Using Relationship Learning Based Super Resolution Algorithm", American Journal of Applied Sciences 11 (3): 475-481, 2014

[10] Taranpreet Singh Ruprah, "Face Recognition Based on PCA Algorithm", Special Issue of International Journal of Computer Science \& Informatics (IJCSI), ISSN (PRINT) : 2231-5292, Vol.- II, Issue-1, 2

[11] Kirby and Sirovich, 1990. Application ofKarhunenLoeve procedure for thecharacterization of human faces. IEEE Trans.pattern analysis and machine intelligence, 12:103-108.

[12] Jonathon Shlens, "A Tutorial on Principal Component Analysis", Systems Neurobiology Laboratory, Ver.2, 2005

[13] M. ParisaBeham and S. Mohamed MansoorRoomi, "Face Recognition Using Appearance Based Approach: 
A Literature Survey", International Conference \& Workshop on Recent Trends in Technology, (TCET) 2012.

[14] Zhujie, Y.L.Y., 1994. Face recognition with Eigen faces. Proc.IEEE Intl. Conf. Industrial Technol.Pp: 434-438.

[15] A.Eleyan and H. Demirel "Face Recognition using Multiresolution PCA" IEEE International Symposium on Signal Processing and Information Technology2007.

[16] Senthilsingh.C and Dr.M.Manikandan, "Design and implementation of an fpga-based real-time very low resolution facerecognition system", International Journal of Advanced Information Science and Technology (IJAIST),November 2012

\section{BIOGRAPHIES}

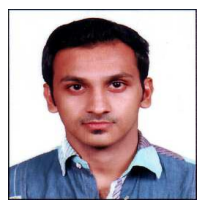

Abin Abraham Oommen received B.Tech degree in Electronics and Communications from University of Kerala, India in 2012 and he is currently pursuing the M.Tech degree in VLSI and Embedded systems at $\mathrm{TocH}$ Institute of Science and Technology,Cochin, India.

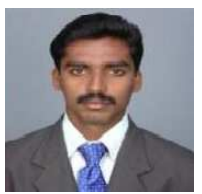

C.Senthil Singh received the B.E.degree in electrical and electronics engineering from the MS University, India,M.Tech in VLSI Design, Currently doing Ph.D.in Information and Communication in Anna University, Chennai, India. His research interest includes VLSI, Image processing. 